\title{
PRINCIPAL BLOCKS AND THE STEINBERG CHARACTER
}

\author{
GERHARD HISS
}

\begin{abstract}
We determine the finite simple groups of Lie type of characteristic $p$, for which the Steinberg character lies in the principal $\ell$-block for every prime $\ell \neq p$ dividing the order of the group.
\end{abstract}

In [1, Corollary 4.4], Bessenrodt, Malle, and Olsson determine the finite simple groups of Lie type having a non-trivial irreducible character which is in the principal $\ell$-block for all primes $\ell$ dividing the group order. Motivated by this and their subsequent investigations on block separations and inclusions [2], Bessenrodt and Zhang asked the following question. Which are the finite simple groups of Lie type of characteristic $p$, such that the Steinberg character is in the principal $\ell$-block for all primes $\ell \neq p$ dividing the group order? In the notation of [1], this asks for those groups $G$ of this class for which the trivial character and the Steinberg character of $G$ are not separated by $\pi(G) \backslash\{p\}$.

Here we answer the question of Bessenrodt and Zhang. It turns out that the answer is generic in the sense that it only depends on the Lie type of the group and not on the underlying characteristic. This genericity suggests that there could be a more uniform proof than the one presented here.

Theorem. Let $G$ be a finite simple group of Lie type of characteristic $p$. Then the Steinberg character of $G$ lies in the principal $\ell$-block of $G$ for all primes $\ell \neq p$ dividing the order of $G$, if and only if $G$ is one of the groups in the following list.

(1) $\operatorname{PSL}_{n}(q)$ with $2 \leq n \leq 4,(n, q) \neq(2,2),(2,3)$.

(2) $\operatorname{PSU}_{n}(q)$ with $3 \leq n \leq 4,(n, q) \neq(3,2)$.

(3) $\operatorname{PSp}_{4}(q), q \neq 2$.

(4) $\mathrm{P} \Omega_{8}^{+}(q)$.

(5) $G_{2}(q), q \neq 2$.

(6) $F_{4}(q)$.

(7) ${ }^{3} D_{4}(q)$.

(8) ${ }^{2} B_{2}(q), q=2^{2 m+1}>2$.

(9) ${ }^{2} G_{2}(q), q=3^{2 m+1}>3$.

(10) ${ }^{2} F_{4}(q), q=2^{2 m+1}>2$.

Date: April 29, 2008.

2000 Mathematics Subject Classification. 20C15, 20 C33.

Key words and phrases. Steinberg character, principal block. 
Proof. The fact that all the groups in the above list satisfy the claimed property can be proved with the help of the Brauer criterion. Generic character tables of the twisted groups of Lie type, at least of their unipotent characters, are available in the literature $[6,18,20,15]$. The character tables of the Chevalley group of type $G_{2}$ are published in $[4,7,8]$. The tables can also be obtained through CHEVIE [12], which in addition provides programs to compute central characters and reduce these modulo the relevant primes. The assertion for the groups of type $F_{4}$ has been verified by Frank Lübeck with the help of his programs producing lists of representatives for the conjugacy classes and the corresponding centralizer orders. For groups of type $F_{4}$ such lists have first been computed by Shinoda and Shoji, and the tables published in $[16,17]$ allow to check the result for these groups by hand.

The claim for the classical groups can be proved with the results of Fong and Srinivasan [9, 10]. For $\ell=2$ (and odd $q$ ) one can use [13] to show that the Steinberg character lies in the principal 2-block.

Let $G$ be a finite simple group of characteristic $p$ not contained in the list of the theorem. We have to show that there is a prime $\ell \neq p$ such that the Steinberg character is not in the principal $\ell$-block. For this purpose we use the Brauer criterion applied to a particular semisimple element of $G$ and a suitable prime $\ell$.

Let $s$ be a semisimple element of $G$ and put $C:=C_{G}(s)$. Then, if $\chi$ is an irreducible character of $G$, the value of the central character corresponding to $\chi$ on the class sum containing $s$ equals

$$
|G: C| \chi(s) / \chi(1) \text {. }
$$

In particular, if $\chi=$ St is the Steinberg character of $G$, the value of (1) equals

$$
\pm|G: C|_{p^{\prime}} .
$$

Subtracting (2) from the corresponding value for the trivial character of $G$ we obtain

$$
\left(|G: C|_{p} \mp 1\right)|G: C|_{p^{\prime}}
$$

In the following, $q$ denotes a power of $p$. If $d$ is a positive integer, we call a prime $\ell$ a Zsigmondy prime for $q^{d}-1$, if $\ell$ divides $q^{d}-1$, but not $q^{i}-1$ for $1 \leq i<d$. Some authors call such an $\ell$ a primitive prime divisor of $q^{d}-1$. A Zsigmondy prime for $q^{d}-1$ exists whenever $d>2$ and $(q, d) \neq(2,6)$ (see [14, Theorem IX.8.3]).

We may as well replace $G$ by a central extension of $G$. We first let $G=G_{n}(q)$ be one of the groups $\mathrm{SL}_{n}(q), \mathrm{SU}_{n}(q), n \geq 5$, or $\mathrm{Sp}_{n}(q), n \geq 6$ even. Let $s \in \mathrm{SL}_{2}(q)$ be a generator of the Coxeter torus, i.e., $s$ has order $q+1$. Then $s$ embeds into $G$ such that $C:=C_{G}(s)$ contains the subgroup $G_{n-2}(q)$ with $p^{\prime}$-index. In the first two cases we have

$$
|G: C|_{p}=q^{2 n-3} \text {. }
$$


In the third case we have

$$
|G: C|_{p}=q^{n-1} \text {. }
$$

In the first and third case we choose a Zsigmondy prime $\ell$ for $q^{n-2}-1$ if $(q, n) \neq(2,8)$, and $\ell=31$ if $(q, n)=(2,8)$. In the second case we choose a Zsigmondy prime $\ell$ for $q^{n-2}-1$ if $n$ is even, and for $q^{2(n-2)}-1$ if $n$ is odd. Then $\ell \nmid\left(|G: C|_{p} \pm 1\right)|G: C|_{p^{\prime}}$.

An excellent reference for the facts used in the following arguments for the orthogonal groups is [19, Chapters 11, 12]. First, let $G=\Omega_{2 m+1}(q)$ with $q$ odd and $m \geq 3$. There is an involution $s \in G$ such that $C:=C_{G}(s)=$ $\Omega_{2 m}^{+}(q)$ or $\Omega_{2 m}^{-}(q)$. Then $|G: C|_{p^{\prime}}=q^{m} \pm 1$ and $|G: C|_{p}=q^{m}$. Let $\ell$ be a Zsigmondy prime for $q^{2 m-2}-1$. Then $\ell \nmid q^{m} \pm 1$, and we are done.

Next, let $G=\Omega_{2 m}^{+}(q) \leq \mathrm{SO}_{2 m}^{+}(q)$ with $m \geq 5$. There is a semisimple element $s \in G$ such that $\Omega_{2 m-4}^{+}(q)$ is contained in $C:=C_{G}(s)$ with $p^{\prime}$-index. Thus

$$
|G: C|=q^{4 m-6}\left(q^{m-2}+1\right)\left(q^{2 m-2}-1\right)\left(q^{m}-1\right) / r
$$

for some $p^{\prime}$-integer $r$. If $(m, q) \neq(8,2)$ we let $\ell$ be a Zsigmondy prime for $q^{m-2}-1$. For $m=8$ and $q=2$ we let $\ell=31$. Then $\ell \nmid|G: C|_{p^{\prime}}\left(|G: C|_{p} \pm 1\right)$.

Finally, let $G=\Omega_{2 m}^{-}(q)$ with $m \geq 4$. There is a semisimple element $s \in G$ such that $\Omega_{2 m-2}^{-}(q)$ is contained in $C:=C_{G}(s)$ with $p^{\prime}$-index. Thus

$$
|G: C|=q^{2(m-1)}\left(q^{m}+1\right)\left(q^{m-1}+1\right) / r
$$

for some $p^{\prime}$-integer $r$. If $(m, q) \neq(5,2)$, let $\ell$ be a Zsigmondy prime for $q^{2 m-4}-1$. If $(m, q)=(5,2)$, let $\ell=7$. Then $\ell \nmid|G: C|_{p^{\prime}}\left(|G: C|_{p} \pm 1\right)$. This completes the proof for the classical groups.

To show that the Steinberg character of an exceptional group $G(q)$ is not contained in the principal block for some prime $\ell$ dividing $|G(q)|$, we use the results of Broué, Malle, and Michel [3, Theorem 5.24, Tables 1, 2]. If $G$ is of type $E_{6},{ }^{2} E_{6}, E_{7}$, and $E_{8}$, respectively, we put $d:=5,10,5$, and 18 , respectively, and let $\ell$ be a Zsigmondy prime for $q^{d}-1$. Then $\ell>d$ and $d$ is the order of $q$ modulo $\ell$. The claim now follows from [3, Tables 1, 2].

Remarks. Let $G$ be one of the non-simple groups belonging to the series of groups listed in the theorem, i.e., $G$ is one of $\mathrm{PSL}_{2}(2) \cong S_{3}, \mathrm{PSL}_{2}(3) \cong$ $A_{4}, \mathrm{PSU}_{3}(2), \mathrm{PSp}_{4}(2) \cong S_{6}, G_{2}(2),{ }^{2} B_{2}(2),{ }^{2} G_{2}(3)$, or ${ }^{2} F_{4}(2)$ (where the characteristic $p$ of $G$ is the one indicated in its name; thus ${ }^{2} G_{2}(3) \cong \mathrm{P}_{2}(8)$ is viewed as a group of characteristic 3$)$. Then $G$ also satisfies the conclusion of the theorem, i.e., the Steinberg character of $G$ lies in the principal $\ell$-block for all primes $\ell \neq p$ dividing $|G|$. This can easily be checked with the Atlas [5] or GAP [11].

Let $N$ be a normal subgroup of $G$, and $\ell$ be prime as above. By a general result on block covering, the constituents of the restriction of the Steinberg character of $G$ to $N$ lie in the principal $\ell$-block of $N$. 
Acknowledgements. I thank Frank Lübeck for checking the Brauer criterion for the exceptional Chevalley groups of type $F_{4}$. Work on this project began during my visit in China in September 2007 financed by the SinoGerman Center for Research Promotion and I thank this institution for their support.

\section{REFERENCES}

[1] C. Bessenrodt, G. Malle, And J. B. Olsson, Separating characters by blocks, J. London Math. Soc. 73 (2006), 493-505.

[2] C. Bessenrodt And J. Zhang, Block separations and inclusions, Adv. Math., to appear.

[3] M. Broué, G. Malle, and J. Michel, Generic blocks of finite reductive groups, Astérisque 212 (1993), 7-92.

[4] B. Chang And R. Ree, The characters of $G_{2}(q)$, in: Symposia Mathematica Vol. 13, pp. 395-413, Academic Press, London, 1974.

[5] J. H. Conway, R. T. Curtis, S. P. Norton, R. A. Parker, and R. A. Wilson, Atlas of Finite Groups, Clarendon Press, Oxford, 1985.

[6] D. I. Deriziotis And G. O. Michler, Character table and blocks of finite simple triality groups ${ }^{3} D_{4}(q)$, Trans. Am. Math. Soc. 303 (1987), 39-70.

[7] H. Enомото, The characters of the finite Chevalley group $G_{2}(q), q=3^{f}$, Japan. J. Math., 2 (1976), 191-248.

[8] H. Enomoto and H. Yamada, The characters of $G_{2}\left(2^{n}\right)$, Japan. J. Math., 12 (1986), 325-377.

[9] P. Fong And B. SRinivasan, The blocks of finite general linear and unitary groups, Invent. Math. 69 (1982), 109-153.

[10] P. Fong And B. SRinivasan, The blocks of finite classical groups, J. reine angew. Math., 396 (1989), 122-191.

[11] The GAP Group, GAP - Groups, Algorithms, and Programming, Version 4.4.10, 2007, (http://www.gap-system.org).

[12] M. Geck, G. Hiss, F. Lübeck, G. Malle, and G. Pfeiffer, CHEVIE-A system for computing and processing generic character tables, AAECC 7 (1996), 175-210.

[13] G. Hiss, The number of trivial composition factors of the Steinberg module, Arch. Math. 54 (1990), 247-251.

[14] B. Huppert and N. Blackburn, Finite Groups II, Springer-Verlag, Berlin, 1982.

[15] G. Malle, Die unipotenten Charaktere von ${ }^{2} F_{4}\left(q^{2}\right)$, Comm. Algebra 18 (1990), $2361-2381$.

[16] K. Shinoda, The conjugacy classes of Chevalley groups of type $\left(F_{4}\right)$ over finite fields of characteristic 2, J. Fac. Sci. Univ. Tokyo Sect. I A Math. 21 (1974), 133-159.

[17] T. ShojI, The conjugacy classes of Chevalley groups of type $\left(F_{4}\right)$ over finite fields of characteristic $p \neq 2$, J. Fac. Sci. Univ. Tokyo Sect. I A Math. 21 (1974), 1-17.

[18] M. Suzuki, On a class of doubly transitive groups, Ann. Math. 75 (1962), 105-145.

[19] D. E. TAYLOR, The Geometry of the Classical Groups, Heldermann Verlag, Berlin, 1992.

[20] H. N. WARD, On Ree's series of simple groups, Trans. Amer. Math. Soc. 121 (1966), $62-89$.

Lehrstuhl D für Mathematik, RWTH Aachen University, 52056 Aachen, GERMANY

E-mail address: gerhard.hiss@math.rwth-aachen.de 